\title{
Administration of Tranexamic Acid as Treatment for Angiotensin Converting Enzyme Inhibitor-Induced Angioedema: A Case Report
}

\author{
Sohun Awsare David Chirikian Joseph Rogers \\ California Northstate University College of Medicine, Elk Grove, CA, USA
}

\section{Keywords}

Angiotensin converting enzyme-inhibitor angioedema - Tranexamic acid · Angiotensin converting enzyme inhibitors · Adverse events

\begin{abstract}
Background

Angiotensin converting enzyme inhibitor-induced angioedema is a major cause of angioedema-related hospitalizations each year. Common swelling predilection sites include the face, periorbital skin, lips, pharynx, larynx, and tongue. Involvement of airway structures could lead to airway obstruction and ultimately death if not treated promptly. A 62-year-old man presented to a rural clinic with a massively swollen tongue that obscured visualization of the retropharynx and mildly swollen lips. The patient stated that he had been taking lisinopril for many years and never had a swelling episode similar to this. The patient was initially administered epinephrine, antihistamine, steroids, and Tranexamic acid. A second dose of Tranexamic acid was administered 30 min later followed by close monitoring. Seven hours after the first administration, the patient had near-complete resolution of tongue swelling and was later discharged. Angioedema treatment is still variable and hospitals in different settings may be able to take advantage of available resources for treatment. Tranexamic acid is a cheap and widely available antifibrinolytic that may be used to treat angiotensin converting enzyme inhibitor-induced angioedema, especially if more specific therapeutics are unavailable.
\end{abstract}




\section{Introduction}

Hypertension is a prevalent disease affecting $>70$ million Americans, or nearly one-third the population [1]. A popular option for treatment has been the use of angiotensin converting enzyme (ACE) inhibitors. They represent just over one-third of all prescriptions written for hypertension in the USA [2]. In addition to their hypertensive benefits, these drugs are also used for the management of other clinical conditions including diabetes mellitus, chronic kidney disease, and heart failure [3]. ACE inhibitors are typically well tolerated by patients and suspending treatment with this agent is rare [4]. ACE inhibitor side effects typically include cough, skin rashes, and hyperkalemia [5]. A less prevalent side effect of ACE inhibitors is angioedema. Angioedema presents as nonpitting, swelling of subcutaneous tissues that typically includes the upper airway, face, tongue, and lips.

Angioedema is the result of mast cell degranulation or activation of kinin formation. The angioedema is induced in 0.1-0.7 percent of recipients with a persistent risk over time [6, 7]. There have been studies that suggest that African Americans are predisposed to a higher incidence of angioedema [8]. In addition to the lips, tongue, and face, angioedema can also affect the larynx and pharynx in which case there may be signs of hoarseness and inspiratory stridor. There have been instances where these clinical symptoms have led to inpatient admissions or ICU care and fatalities have been reported $[9,10]$. The onset of angioedema is typically within the first month of beginning the treatment; however, angioedema has been documented to occur from hours to years after initiation of the drug [7]. Here, we describe the management of a patient that presents with ACE inhibitor-induced angioedema and subsequent discharge from care.

\section{Case Presentation}

A 62-year-old male presented to the emergency department with a chief complaint of a swollen tongue. The patient reported waking up with a massively swollen tongue (Fig. 1). He reported discomfort in the tongue but otherwise noted the tongue is not painful. The patient's lips were mildly swollen, while the tongue was significantly enlarged occupying most of the oropharynx and obscuring full visualization of the retropharynx. The tongue extended out of the mouth. The uvula was visible and was hanging midline with no uvular edema. The patient's airway was widely patent, and the patient had no drooling, no difficulty handling his secretions, and no stridor. The patient reported a history of hypertension, chronic kidney disease, diabetes, chronic pain, hyperlipidemia, and obesity. Of note, this patient has taken lisinopril for years and has never had angioedema or other adverse reactions to the medication until now. He has a family history of hypertension and type 2 diabetes but denied family members with similar complications and presentation in the past. He denied the use of any other drugs and denied other toxic exposures. The patient denied fevers, chills, sweats, coughing, wheezing, dyspnea, chest pain, and rash. He presented as hypertensive with a blood pressure of $153 / 101$. His pulse was 95 beats per minute, and his respiratory rate was 20 breaths per minute. His mental status was clear, and the patient was alert, calm, and cooperative. The patient had no other noted edema. The patient was administered epinephrine $0.5 \mathrm{mg}$ intramuscularly, antihistamine $50 \mathrm{mg}$ intravenously, steroids $125 \mathrm{mg}$ intravenously, and Tranexamic acid 1,000 mg. In 30 min intervals, the patient was repeatedly checked. Thirtythree minutes after initial presentation, there was less tongue swelling, improved phonation, and decreased tongue swelling. The patient was administered a second dose of Tranexamic acid of $1,300 \mathrm{mg}$ at $10 \mathrm{mg} / \mathrm{kg}$. At $7 \mathrm{~h}$ and $3 \mathrm{~min}$ after the presentation, there was near resolution of tongue swelling, and the patient was able to swallow a can of juice without difficulty.

\section{Karger'}


Fig. 1. Oral cavityoral cavity upon presentation to the emergency department with notably enlarged tongue.

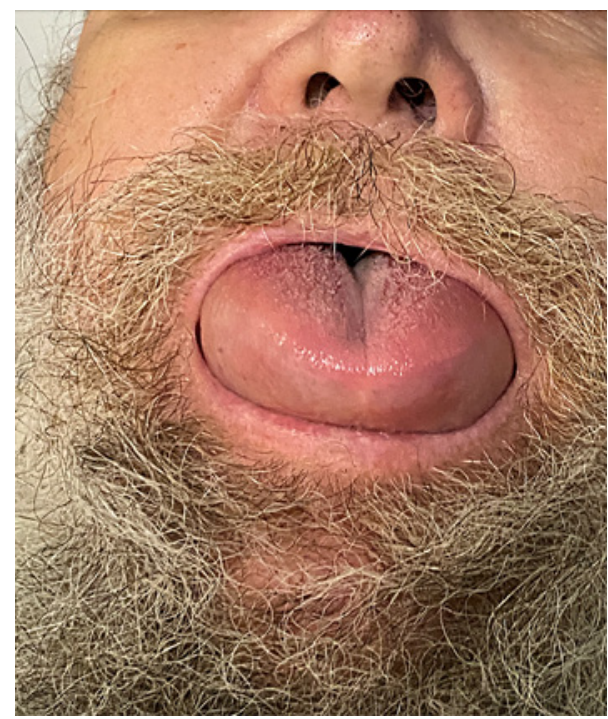

The patient's airway was fully patent, and his phonation had improved. The patient was stable for discharge and instructed to return if similar symptoms ever presented.

\section{Discussion/Conclusion}

Angioedema is defined as rapid, nonpitting swelling commonly affecting the face, periorbital skin, lips, larynx, pharynx, tongue, and in some cases, the gastrointestinal tract [11]. Swelling of the tongue, larynx, and pharynx is especially problematic due to the increased risk of obstruction to the airway. In severe cases, airway integrity can be lost, leading to death. Angioedema can further be categorized as hereditary, which is associated with a C1 inhibitor deficiency and mutations on chromosome 11, allergic angioedema, which can be caused by food allergies, and drug-induced angioedema, which can arise from the use of nonsteroidal anti-inflammatory drugs or ACE inhibitors [11]. Deaths due to hereditary angioedema are rare and have decreased from 1979 to 2010, while deaths by nonhereditary angioedema have since risen [12]. ACE inhibitors are thought to be one of the major causes of nonhereditary angioedema leading to hospitalization and may comprise $30 \%$ of all angioedema cases presenting to the emergency room [9]. Furthermore, one large multihospital study determined that the rate of ACE inhibitor-induced angioedema annually is approximately 0.7 per 10,000 emergency room visits [9].

The mechanism of action in ACE inhibitor-induced angioedema is linked to decreased bradykinin degradation and the kallikrein-kinin system [13]. ACE is an important enzyme associated with bradykinin breakdown. The accumulation of excess bradykinin is thought to cause plasma extraversion, vasodilation, and ultimately, angioedema. Moreover, hereditary angioedema is also a bradykinin-mediated condition whereas allergic angioedema is a histamine-mediated response [13]. Distinguishing between a bradykinin-mediated angioedema and a histamine-mediated angioedema is not always simple and must be carefully considered and diagnosed in the clinic. The treatment for histamine-mediated angioedema involves administration of antihistamines, glucocorticoids, epinephrine, and acute airway support if needed. The treatment for bradykinin-mediated angioedema, such as ACE inhibitorinduced angioedema, involves discontinuation of the causative drug, steroids, antihistamines, epinephrine, intravenous fluids, and airway support measures, such as endotracheal 
intubation $[9,14]$. Acute management of hereditary angioedema may include fresh frozen plasma and intubation if suspected compromise of the airway [15]. Additional therapies for bradykinin-induced angioedema include plasma-derived C1 inhibitors (Berinert and Cinryze), recombinant C1 Inhibitors (Ruconest), and kallikrein inhibitor (Icatibant/Kalbitor) [16].

Tranexamic acid is a commonly used antifibrinolytic drug that is used to treat excessive bleeding associated with surgeries, pregnancies/menorrhagia, clotting disorders, and trauma [17]. Although its use is controversial, Tranexamic acid is also used acutely and prophylactically to treat hereditary angioedema [18]. It binds plasminogen and inhibits the formation of plasmin, a key step typically regulated by $\mathrm{C} 1$ inhibitor, which is a protein that is deficient in hereditary angioedema patients. A recently published case report showed that Tranexamic acid may be used to treat ACE inhibitor-induced angioedema [19]. Furthermore, an efficacy study showed that Tranexamic acid is a cheap and widely available drug that may yield positive treatment outcomes in emergency rooms that lack more specific ACE inhibitorinduced angioedema therapeutics, such as Icatibant and C1INH concentrate [20]. More research needs to be conducted regarding the efficacy of Tranexamic acid as a first-line treatment option for ACE inhibitor-induced angioedema. However, if more specific treatment options in the acute setting are unavailable, such as in a rural-based hospital, Tranexamic acid should be considered as a potential therapy.

\section{Acknowledgements}

We thank all members of our research team for their assistance with this project.

\section{Statement of Ethics}

The patient described in this manuscript has given written, informed consent to publish his case, radiographic images, and pathological reports. The research in this manuscript was conducted ethically in accordance with the World Medical Association Declaration of Helsinki. The manuscript is exempt from Ethical Committee approval as this is an individual case report that does not impose study designs or other parameters and does not have any negative consequences for the patient.

\section{Conflict of Interest Statement}

The authors have no conflicts of interest to declare.

\section{Funding Sources}

No funding sources to be reported.

\section{Author Contributions}

Sohun Awsare and David Chirikian equally contributed to reviewing the patient chart, conducting a literature review, and drafting the manuscript. Dr. Joseph Rogers reviewed literature and revised the manuscript. 


\section{Availability of Data and Material}

Data sharing not applicable-no new data generated.

\section{References}

1 Benjamin EJ, Muntner P, Alonso A, Bittencourt MS, Callaway CW, Carson AP, et al. Heart disease and stroke statistics-2019 update: a report from the American Heart Association. Circulation. 2019 Mar 5;139(10):e56528. Erratum in: Circulation. 2020 Jan 14;141(2):e33.

2 Ma J, Lee KV, Stafford RS. Changes in antihypertensive prescribing during US outpatient visits for uncomplicated hypertension between 1993 and 2004. Hypertension. 2006;48:846.

3 Weir MR. Effects of renin-angiotensin system inhibition on end-organ protection: can we do better? Clin Ther. 2007 Sep;29(9):1803-24.

4 Mahmoudpour SH, Asselbergs FW, Souverein PC, de Boer A, Maitland-van der Zee AH. Prescription patterns of angiotensin-converting enzyme inhibitors for various indications: a UK population-based study. Br J Clin Pharmacol. 2018;84(10):2365-72.

5 Bavishi C, Ahmed M, Trivedi V, Khan AR, Gongora C, Bangalore S, et al. Meta-analysis of randomized trials on the efficacy and safety of angiotensin-converting enzyme inhibitors in patients $\geq 65$ years of age. Am J Cardiol. 2016 Nov 1;118(9):1427-36. Epub 2016 Aug 22.

6 Toh S, Reichman ME, Houstoun M, Ross Southworth M, Ding X, Hernandez AF, et al. Comparative risk for angioedema associated with the use of drugs that target the renin-angiotensin-aldosterone system. Arch Intern Med. 2012;172:1582.

7 Banerji A, Blumenthal KG, Lai KH, Zhou L. Epidemiology of ACE Inhibitor Angioedema utilizing a large electronic health record. J Allergy Clin Immunol Pract. 2017;5:744.

8 Brown NJ, Ray WA, Snowden M, Griffin MR. Black Americans have an increased rate of angiotensin converting enzyme inhibitor-associated angioedema. Clin Pharmacol Ther. 1996 Jul;60(1):8-13.

9 Banerji A, Clark S, Blanda M, LoVecchio F, Snyder B, Camargo CA Jr. Multicenter study of patients with angiotensin-converting enzyme inhibitor-induced angioedema who present to the emergency department. Ann Allergy Asthma Immunol. 2008 Apr;100(4):327-32.

10 Dean DE, Schultz DL, Powers RH. Asphyxia due to angiotensin converting enzyme (ACE) inhibitor mediated angioedema of the tongue during the treatment of hypertensive heart disease. J Forensic Sci. 2001 Sep;46(5): 1239-43.

11 Kaplan AP. Angioedema. World Allergy Organ J. 2008;1(6):103-13.

12 Kim SJ, Brooks JC, Sheikh J, Kaplan MS, Goldberg BJ. Angioedema deaths in the United States, 1979-2010. Ann Allergy Asthma Immunol. 2014 Dec;113(6):630-4. Epub 2014 Oct 3.

13 Kaplan AP, Joseph K, Silverberg M. Pathways for bradykinin formation and inflammatory disease. J Allergy Clin Immunol. 2002 Feb;109(2):195-209.

14 Kostis WJ, Shetty M, Chowdhury YS, Kostis JB. ACE Inhibitor-induced angioedema: a review. Curr Hypertens Rep. 2018 Jun 8;20(7):55.

15 Moellman JJ, Bernstein JA, Lindsell C, Banerji A, Busse PJ, Camargo CA, et al. A consensus parameter for the evaluation and management of angioedema in the emergency department. Acad Emerg Med. 2014;21(4): 469-84.

16 Long BJ, Koyfman A, Gottlieb M. Evaluation and management of angioedema in the emergency department. West J Emerg Med. 2019;20(4):587-600.

17 Cai J, Ribkoff J, Olson S, Raghunathan V, Al-Samkari H, DeLoughery TG, et al. The many roles of tranexamic acid: an overview of the clinical indications for TXA in medical and surgical patients. Eur J Haematol. 2020;104(2): 79-87.

18 Horiuchi T, Hide M, Yamashita K, Ohsawa I. The use of tranexamic acid for on-demand and prophylactic treatment of hereditary angioedema: a systematic review. J Cutan Immunol Allergy. 2018;1(4):126-38.

19 Wang K, Geiger H, McMahon A. Tranexamic acid for ACE inhibitor induced angioedema: a case report. Am J Emerg Med. 2020 Oct 21. Epub ahead of print.

20 Beauchêne C, Martins-Héricher J, Denis D, Martin L, Maillard H. [Tranexamic acid as first-line emergency treatment for episodes of bradykinin-mediated angioedema induced by ACE inhibitors]. Rev Med Interne. 2018 Oct;39(10):772-6. Epub 2018 May 4. French. 